\title{
El diseño complejo
}

\section{Resumen}

El diseño no solo es una disciplina difícil de entender a partir de todas las definiciones que en torno a él se han planteado, sino que tal entendimiento se complica más aún cuando este se ejerce en términos profesionales. El concepto disciplinar que sustenta el presente texto define el diseñar como una ecualización de variables con resultado de forma, por lo que asociar el diseño profesional exclusivamente a la forma o pretender restringirlo al ámbito técnico de la producción resulta inadecuado, toda vez que lo realmente trascendente es la cuestión del valor que adquieren los objetos para un perfil de usuario determinado. Luego si en los tiempos del consumo globalizado las valoraciones han cambiado, ello requiere que la formación de los diseñadores profesionales de nivel superior también cambien en sus métodos y contenidos de manera que permitan comprender los fenómenos sociales contemporáneos como un primer paso para la proposición de nuevas realidades.

En estos términos es que frente a un escenario que se estima dominado por la especulación económica y la desesperanza social, el diseño adquiere valor cuando contribuye a reposicionar la condición emocional y estética del ser humano.

\section{Diseño y diseñadores}

En una ocasión, y en el marco de la mantención de mi automóvil, acudí a un local de reparación de frenos cuyo servicio prometía ser rápido al punto que se podía esperar mientras el trabajo se realizaba. Tal vez en el ánimo de que la espera no fuese tan tediosa, el encargado del local, vistiendo una impecable cotona blanca, se me acerca e inicia una conversación que comienza al preguntar: "¿A qué se dedica usted?”, yo le respondí: soy diseñador industrial. Como tenía tiempo, además de estar imbuido en un espíritu pedagógico y de promoción de la disciplina, me sentí dispuesto a explayarme en la respuesta siguiente cuando me preguntara respecto a qué hacían los diseñadores industriales; sin embargo, y para mi sorpresa, el comentario del encargado fue: “ $\mathrm{A}$ h, yo también soy diseñador!”, y 
señalando hacia un costado del local en que estaban emplazadas tres plataformas de las usadas para levantar los vehículos y retirar las ruedas, agregó: "Estas tres unidades las diseñé yo, y las tres son diferentes porque las he ido perfeccionando... incluso hemos vendido algunas". Luego, y dirigiendo la vista hacia las plataformas, continuó: "Claro, hay que reconocer que la parte de los comandos no me ha resultado mucho, porque los pasan a llevar y usted ve como están, todos chuecos". Efectivamente las llaves que estaban a ras de suelo lucían torcidas, algunas con sus manillas quebradas, producto de ser aplastadas por las ruedas de los vehículos que ingresaban a las plataformas, ya que en general las llaves estaban ubicadas en puntos ciegos para la posición del conductor. Además las manillas de las llaves correspondían a modelos de acción manual; sin embargo, los operarios las accionaban con el pie para no tener que agacharse cada vez que requerían accionarlas.

"Lo que pasa, continuó el encargado, es que hace un tiempo descubrí que la presión de una cámara de camión tiene la fuerza suficiente para levantar un auto, y como nosotros necesitamos sólo que las ruedas -del auto- se despeguen del suelo, con la carrera que produce el inflado de la cámara alcanza”. Al agacharme y mirar bajo las plataformas vi que efectivamente no había un sistema hidráulico debajo, sino que una cámara de camión ubicada en el medio, cuya deformación por el inflado levantaba todo el sistema. Y agregó: "La primera, esa de allá -señalando la que estaba más al rincón- me quedó sobredimensionada, así que en los modelos siguientes, cada vez le saco más material y con eso bajo el costo". Efectivamente lo que señalaba como el primer modelo estaba compuesto por grandes piezas de plancha de acero diamantado, en tanto el último se acercaba bastante más a un esqueleto.

Como comentario aparte y casi a modo de justificación señaló: "Es que hace 27 años que trabajo en esto y uno siempre le está dando vueeeltas. Uno nunca se desconecta totalmente de la pega”.

Entre los posteriores comentarios del encargado acerca de los modelos de autos, la calidad del servicio y el fútbol, me quedé pensando, buscando diferencias y coincidencias entre su modo de diseñar y el mío.

\section{El diseño espontáneo}

El diseño espontáneo se trata de una actividad configurativa que se vincula directamente con la condición biológica y psicológica del hombre, la cual lo impulsa a crear y perfeccionar sus técnicas de trabajo para construir y mantener el entorno artificial que requiere la implementación de su modo de vida.
El diseño espontáneo, generalmente se da en la solución de problemas en el ámbito personal, por lo que el diseñador no percibe remuneración a cambio de su trabajo. Además no se trata de una actividad permanente, sino que es una actividad esporádica que se inserta como una tarea más dentro de procesos productivos genéricos. En esos términos viene a lugar la afirmación en torno a que todo es diseño y todos somos diseñadores, porque en el fondo la configuración de soluciones es una cuestión cotidiana, cuyos estándares de solución van a la par con el desarrollo cultural de quien lo realiza.

La característica de este modo de diseñar es que el diseñador frecuentemente es parte del problema, luego, tanto los temas abordadas como las argumentaciones de diseño se restringen a las condiciones propias de las labores cotidianas en que se desenvuelve el diseñador, en tanto sus estándares de solución son expresión de los dominios alcanzados en dichas áreas. Ello hace que el diseño espontáneo en realidad no sea tan espontáneo, ya que en él trasciende la memoria, la tradición y la identidad de quien diseña, imprimiendo en sus oficios los valores prácticos y simbólicos propios de su condición sociocultural. En esta dinámica el diseño espontáneo no trasciende y al parecer tampoco pretende trascender a otros ámbitos diferentes de aquel donde se generó la actividad, por lo que la utilidad, inteligibilidad y agradabilidad del producto resulta a la medida del propio diseñador que también es el usuario.

\section{El diseño profesional}

A diferencia de lo espontáneo, lo profesional está referido a una facultad u oficio que alguien ejerce a cambio de una retribución. Retribución que se funda en un sistema económico donde se paga por lo que se desea y que por algún motivo no se puede lograr de manera autónoma, por esta vía, las destrezas y el conocimiento se transforman en mercancías transables cuyo precio aumenta en la medida que el producto generado representa un valor para otras personas y por el cual están dispuestos a pagar.

La profesionalización transforma cualquier actividad que se desarrolla en tales condiciones en un servicio, donde unas personas que actúan en función de otras contribuyen a mejorar sus condiciones operativas a cambio de una retribución. El servicio como tal es un intangible, cuyo beneficio se aprecia cuando la idea se hace operable a través de acciones y elementos concretos que dan lugar a la experiencia de uso. En el proceso es posible distinguir el momento en que la idea del servicio es concebida y separarla de la etapa en que tal idea entra en producción para materializar los elementos que permitan implementar el servicio. 
En el caso del diseño profesional, el diseñador no necesariamente participa de forma física de la producción, pero obligatoriamente -de alguna manera inevitable- ha de conectarse con ella como una manera de fluidizar el proceso para que el usuario llegue a realizar la acción que ayudará a mejorar sus condiciones operativas cotidianas según su modelo de vida. Luego el servicio no consistirá en haber contribuido a generar un producto, sino en el haber aportado -dentro del marco ideológico del usuario- al mejoramiento de la condición operativa mediante el uso de uno o un sistema de productos. ¿Cuánto estima el usuario que la mejoró?, he ahí el valor del producto. Agregar valor a un producto no consiste sólo en agregar trabajo en su elaboración, sino incorporarle un sentido y carácter, de manera que sea interesante para los usuarios, ya que en definitiva el valor real radica en el uso y no en el costo de producción, luego, el valor no está determinado por quien produce, sino por quien consume $(\mathrm{I})$.

En este sentido, la gran diferencia entre el diseño profesional y el diseño espontáneo es que este último trabaja con el valor que otorga el propio diseñador, que a su vez también es el usuario. Por su parte, la paradoja del diseñador profesional es que, no obstante ser usuario en tanto parte de la sociedad, debe desdoblarse y transformar el uso en objeto de estudio tomando distancia de su condición intrínseca de usuario.

Luego, la señal distintiva entre lo profesional y lo espontáneo no está en los niveles de elaboración, los cuales pueden ser muy buenos o muy malos en ambos casos, sino en la capacidad de trascender del propio deseo y otorgar un buen servicio. Cabe señalar que brindar un servicio, parte por ponerse en el lugar del usuario, sin pretender reemplazarlo o imponerle condiciones ajenas a su voluntad. Para el diseñador profesional que trabaja bajo el concepto de servicio, asegurar que las cosas funcionen no es tema, porque eso es lo mínimo que se puede esperar de un objeto; un ventilador que no ventila no alcanza la categoría de ventilador, luego no existe ni siquiera como un mal ventilador. Lo que determina la diferencia entre uno y otro modelo no está en si ventilan o no, sino en la manera de hacerlo y lo gratificante de la experiencia que se deriva de dicha modalidad a la luz de los deseos del usuario.

Un determinado aparato es una solución adecuada al problema de la ventilación si es que ventila y eso generalmente está resuelto desde la ingeniería. En el diseño profesional lo adecuado va acompañado de la excelencia, es decir, que las cosas, además de responder a un propósito central, lo hacen de una manera agradable para el usuario. En este sentido el quehacer del diseñador se aleja del régimen cuantitativo que rige la solución de ingeniería para internarse en el mundo de las cualidades y los conceptos de uso, un mundo de percepciones marcado profundamente por la cultura, la subjetividad y las emociones.

Por los mismos motivos anteriores, en el diseño-servicio la forma no tiene sentido en sí misma ni acapara el protagonismo en la acción, muy por el contrario, asume un rol en función de la satisfacción del usuario. En este punto, cabe establecer un símil entre el objeto de diseño y un "mesero", ya que ambos son invisibles durante sus prestaciones gracias a la fluidez de su desempeño, sin embargo ambos han de generar confianza, credibilidad y admiración cada vez que se visibilizan.

Es aquí cuando aparecen los aspectos perceptivos, propios del diseño, cuya elaboración se traduce en estándares de servicio que se encumbran en el ámbito de la excelencia y cuyos indicadores no son una resultante azarosa del proceso, sino un objetivo establecido desde el principio y cuyo logro está condicionado por el conocimiento, bagaje, riesgo y particularmente el criterio de quien diseña.

Comparando las características entre el diseño espontáneo y el Esquema desarrollado diseño profesional, cabe consignar cinco diferencias fundamentales: por el autor

\section{DP}

El diseñador profesional no es parte del problema, por $\quad$ El diseñador espontáneo es parte del problema ya el contrario, él problematiza para poder comprender que este se da en su ámbito cotidiano de trabajo. la situación.

\begin{tabular}{l|l|}
\hline Trabaja con el valor que asignan otros. & Trabaja con el valor que asigna él mismo. \\
\hline $\begin{array}{l}\text { Como agente externo al contexto del problema } \\
\text { puede asociar información y definir diferentes } \\
\text { estándares de solución. }\end{array}$ & $\begin{array}{l}\text { Como parte del problema y del contexto la } \\
\text { información está restringida a lo que arroja su } \\
\text { práctica cotidiana. }\end{array}$ \\
\hline $\begin{array}{l}\text { Su preocupación está en el uso, ya que el } \\
\text { funcionamiento se importa generalmente desde la } \\
\text { ingeniería. }\end{array}$ & $\begin{array}{l}\text { Su preocupación está en el funcionamiento y el } \\
\text { uso surge desde la práctica. }\end{array}$ \\
\hline $\begin{array}{l}\text { Su formación teórico-práctica enfatiza el acceso y } \\
\text { el análisis de información proveniente de diversos } \\
\text { escenarios. }\end{array}$ & $\begin{array}{l}\text { Su formación es de oficio y se construye } \\
\text { generalmente a partir de un escenario único. }\end{array}$ \\
\hline
\end{tabular}
escenarios

\section{La complejidad del producto}

En primer lugar cabe señalar que la idea de complejo, en este caso, está asociada a las acepciones de diverso y conjunto. Vale decir, está referida a la cantidad de variables consideradas para generar un producto. $\mathrm{Al}$ respecto no basta señalar que se presentan en torno a la usabilidad, la producción y la comercialización, sino que es necesario reconocer los subgrupos contenidos en cada uno de estos conjuntos tipológicos y resolver los roces que se producen entre lo global y lo 
local, entre la ciencia y el hábito, entre lo objetivo y lo subjetivo, entre la tecnología y el medio ambiente, entre lo político y lo económico, entre el valor y el precio.

El producto no es diseño sino una consecuencia de él. Diseño es un proceso de ecualización de variables en función de un propósito, bajo un determinado criterio de realidad, cuyo resultado es la forma. Luego la complejidad del producto no está en lo intrincado de sus procesos productivos, sino en su posibilidad de responder positivamente a todas las interpelaciones a que estará expuesto desde el momento de su concepción, su producción, su uso y su destino al final de su vida útil.

Definir cuándo un producto es complejo y cuándo no lo es, o tratar de establecer desde dónde el producto comienza a complejizarse, podría resultar inoperante dado que en definitiva todo lo construido conlleva algo de complejidad; sin embargo, y expuesto arbitrariamente, un producto empieza a complejizarse cuando las variables presentes exceden un ámbito único de consideraciones y se amplían hacia dominios cada vez más diversos.

En este sentido, el caso relatado al comienzo de este texto, correspondería a un diseño de muy baja complejidad, por cuanto todo el proceso se resuelve solo en el ámbito de la mecánica de funcionamiento, pero no contempla los aspectos de usabilidad, de estructura y de costos, ello independientemente de la tecnología utilizada, la cual puede ser compleja, pero donde cabe consignar que la complejidad corresponde a la tecnología, pero no al proceso de diseño ni al producto, ya que este no responde al ser interpelado desde un criterio de realidad que exceda el ámbito de la mecánica.

La experiencia de un usuario inserto en una sociedad compleja, invariablemente complejiza los requerimientos del producto, porque tal complejidad a la postre se transforma en un estilo de vida en que las interrelaciones se cruzan innumerablemente y en diversos sentidos dando lugar a una trama que además de densa varía según las circunstancias. Al respecto, cabe señalar que el diseño profesional industrial nace en medio del paradigma de la industrialización y el sistema económico liberal, por tanto, su primer compromiso es con la fábrica y las ventas, de ahí que en las economías basadas en la manufactura, el diseño industrial se transforma en un factor estratégico que otorga competitividad al desarrollo tecnológico.

Sin embargo, la venta no se materializa en el ámbito de las máquinas, sino en el mundo de las personas, por tanto los esfuerzos por empatizar con el que compra se multiplican ya que en definitiva es él quien le da movilidad al negocio. Estos esfuerzos han desatado estrategias variadas, enfatizando los aspectos prácticos del producto, las cualidades de marca, las disponibilidades tecnológicas, el precio o los aspectos estéticos, afirmando que en la práctica "lo feo no se vende" (2), todo ello bajo el entendido de que el motivo de las personas para incorporar nuevos objetos a su vida cotidiana, responde fundamentalmente al deseo de mantener una opción de vida, cuyo devenir termina caracterizando el entorno habitable propio de una civilización (3). Ello pone de manifiesto que en el estado cultural del mundo globalizado, más importante que la solución de los problemas del habitar, que ya están solucionados de lo contrario no existiríamos como especie, lo realmente importante son los estándares de servicio, es decir, la manera de implementar las acciones cotidianas, estructurando un sistema que define las expectativas, los estilos y la calidad de vida (4).

Además del hacer, el modo de hacer implica una experiencia que trasciende los aspectos funcionales prácticos, comprometiendo el mundo subjetivo de cada usuario. Retirar el polvo con una escoba o una aspiradora tiene como diferencia las circunstancias que se desprenden de los modos operatorios de cada aparato, los cuales definen requerimientos físicos e intelectuales para el uso, generando con ello un resultado estético.

Una buena experiencia de uso sin duda tiene que ver con la agradabilidad; sin embargo, no existe un parámetro único asociado a la agradabilidad, ya que más allá de lo estrictamente biológico, esto depende de la cultura. No obstante, cabe considerar que el humano es un ser social y estético, luego, la agradabilidad tiene que ver con autovaloración y con estimación de belleza. Por contraposición, una mala experiencia de uso es aquella que dentro de un modelo cultural determinado somete al ridículo, a la ininteligibilidad o a la fealdad(5).

La afirmación se funda en la premisa de que todas las necesidades humanas nacen desde la cultura, por tanto su satisfacción también es un asunto cultural. Según Basalla, las necesidades surgen desde la manera de relacionarse con el entorno, y en estos términos la agradabilidad se transforma en un medio de autoprotección que actúa sobre la base de los patrones subjetivos construidos por cada individuo. Lo que no resulta agradable en principio se rechaza y lo que se percibe agradable en una relación no solo se acepta, sino que define la manera en que esa relación se llevará en el futuro.

\section{Los requisitos de formación}

No obstante los cambios experimentados en el ámbito de la producción y el consumo, los diseñadores industriales, con mayor o menor grado de humanidad, siguen fieles a la misión que se les encomendó desde un principio: generar valor. Es decir, configurar productos por 
los cuales valga la pena pagar, contribuyendo de paso a la definición de estándares de vida.

Primero fueron las artes aplicadas intentando humanizar el producto de las máquinas, luego el hito de la Bauhaus tratando de generar algo parecido a un artesano docto en las artes fabriles y más tarde, la escuela de Ulm que se aleja un tanto de la condicionante productiva y comienza a valorar el producto según los méritos propios del objeto. De ahí en adelante, las corrientes se multiplican siguiendo el ritmo de cambio en las visiones de mundo que se producen en las sociedades desarrolladas, generando una estela de seguidores en los sectores acomodados de las sociedades periféricas, cuya opción es vivir "a la europea". Sin embargo, el fenómeno de la globalización genera una serie de hibridaciones culturales producto de la tensión entre lo local y, particularmente, el modelo económico global que se filtra a todas las dimensiones de la vida actual en todo el mundo.

En este contexto, el diseño complejo -aquel en que confluyen variables de diferente naturaleza, para ser desarrollado requiere capacidades que permitan analizar y manejar dichas variables, lo que supone dominios conceptuales y metodológicos en las áreas: política, analítica, creativa, técnica y evaluativa del proyecto. El diseño complejo no es asociable a formas complejas, muy por el contrario, se refiere a formas simplificadas (6) en cuya resolución influyen argumentaciones provenientes de diferentes ámbitos contenidas en la situación que da origen a la intervención de diseño.

Respecto de los niveles formativos, en primer, lugar está el técnico cuyas competencias se concentran en la operación de equipos o herramientas, por lo que su formación está más cerca de una capacitación en el uso de equipos o dispositivos. Luego está el nivel de tecnólogo, el cual corresponde a un nivel técnico avanzado encargado de materializar la viabilidad productiva del proyecto. Su quehacer se acerca a una visión ingenieril, en tanto su preocupación se centra en cómo resolver prácticamente la producción, por tanto es experto en materiales y procesos.

El nivel superior del diseño lo constituye el diseñador profesional, el cual si bien ha de manejar criterios de producción enfatiza su quehacer en la forma y los aspectos que la condicionan y justifican desde el ámbito de la usabilidad. Si bien el diseñador está en contacto con la industria, este es un medio para producir, pero que no determina por sí mismo el qué y el por qué producir ni la forma que tendrá lo producido.

En este sentido el diseño complejo corresponde a un nivel de desempeño superior, inscrito en una concepción sistémica de la realidad, por lo que reconoce factores diversos que responden a un concepto de calidad total en el producto. Luego, su formación más que técnica se sitúa cercana al arte y las ciencias sociales, enfatizando los aspectos simbólicos y la percepción estética.

Sin duda que no es posible desconocer que en la historia del diseño profesional está representado el desarrollo tecnológico y cultural de la humanidad, lo que ha llevado a la disciplina a recorrer caminos que hoy carecen de sentido, pero que en su momento fueron adecuados. Es en relación a este punto que cabe la detención para analizar qué será lo adecuado no sólo para hoy, sino principalmente para los años venideros en que ya se vislumbra a las ciencias económicas entrando en conflicto con las ciencias sociales y de la tierra, y en el punto de máxima fricción las naciones se debaten entre la especulación y la desesperanza.

El diseño profesional universitario enfrenta un gran desafío particularmente en la universidad pública, la que se entiende no ha de caer en la especulación económica ni en la desesperanza y cortar en tajo profundo para lograr cambios relevantes.

En el diseño-servicio se trabaja la forma; sin embargo, el compromiso es con las personas y sus experiencias de vida y al parecer -según el estado de cosas- es necesario no hacer tantos esfuerzos por figurar en el mundo del consumo, sino iniciar un recorrido off road para potenciar la condición humana y estética del hombre, como parte fundamental del estilo de vida.

En este escenario se hace necesario resistir la tentación de asumir la técnica como elemento neutro con sentido en sí mismo y sin interés político, luego libre de controversia, para introducirse de lleno en las problemáticas del hombre, proponiendo alternativas de enfoque disciplinar fundadas, por ejemplo, en las características más básicas del ser humano, como son los ritmos operativos que se desprenden de su condición biológica y sus patrones perceptivos que se desprenden de su cultura. Sin duda, este es un camino difícil y arriesgado, pero ¿qué sentido tiene el diseño profesional si se le somete a lo establecido y se le restringe el vuelo en materia de riesgo e innovación? 
1. Este aspecto es el que desde una visión mercantilista da pie a la especulación cuando el intermediario maneja el valor que los consumidoresle atribuyen a los productos independientemente de los costos de producción.

2. Raymond Loewy. 1955.

3. Basalla, citado por Fernando Martin Juez en: Contribuciones para una antropología del diseño. pp.52.

Basalla, 1991. Citado por Fernando Martin Juez en: Contribuciones para una antropología del diseño. pp.53. Presentada en El árbol del conocimiento.

4. Según Maturana, el hombre, como estructura biológica, responde emocionalmente a los estímulos provenientes del entorno, la racionalidad surge posteriormente al reflexionar acerca de la experiencia vivida determinando si es razonable, adecuada 0 conveniente. En estos términos la agradabilidad se transforma en un medio de autoprotección que actúa sobre la base de patrones subjetivos construidos por cada individuo. Lo que no resulta agradable en principio se rechaza y lo que se percibe agradable en una relación no solo se acepta, sino que define la manera en que esa relación se llevará en el futuro.

5. Los planteamientos de Maturana, Norman, Zimerman, Sennet y Lipovetski indican que los modos de vida se constituyen perceptivamente a partir de la agradabilidad de los entornos en términos emocionales, la reflexión acerca de su conveniencia es posterior y la transformación de la experiencia en hábitos, es en definitiva la expresión concreta de la cultura.

6. En definitiva, esta también es una variable de costo en la etapa de producción

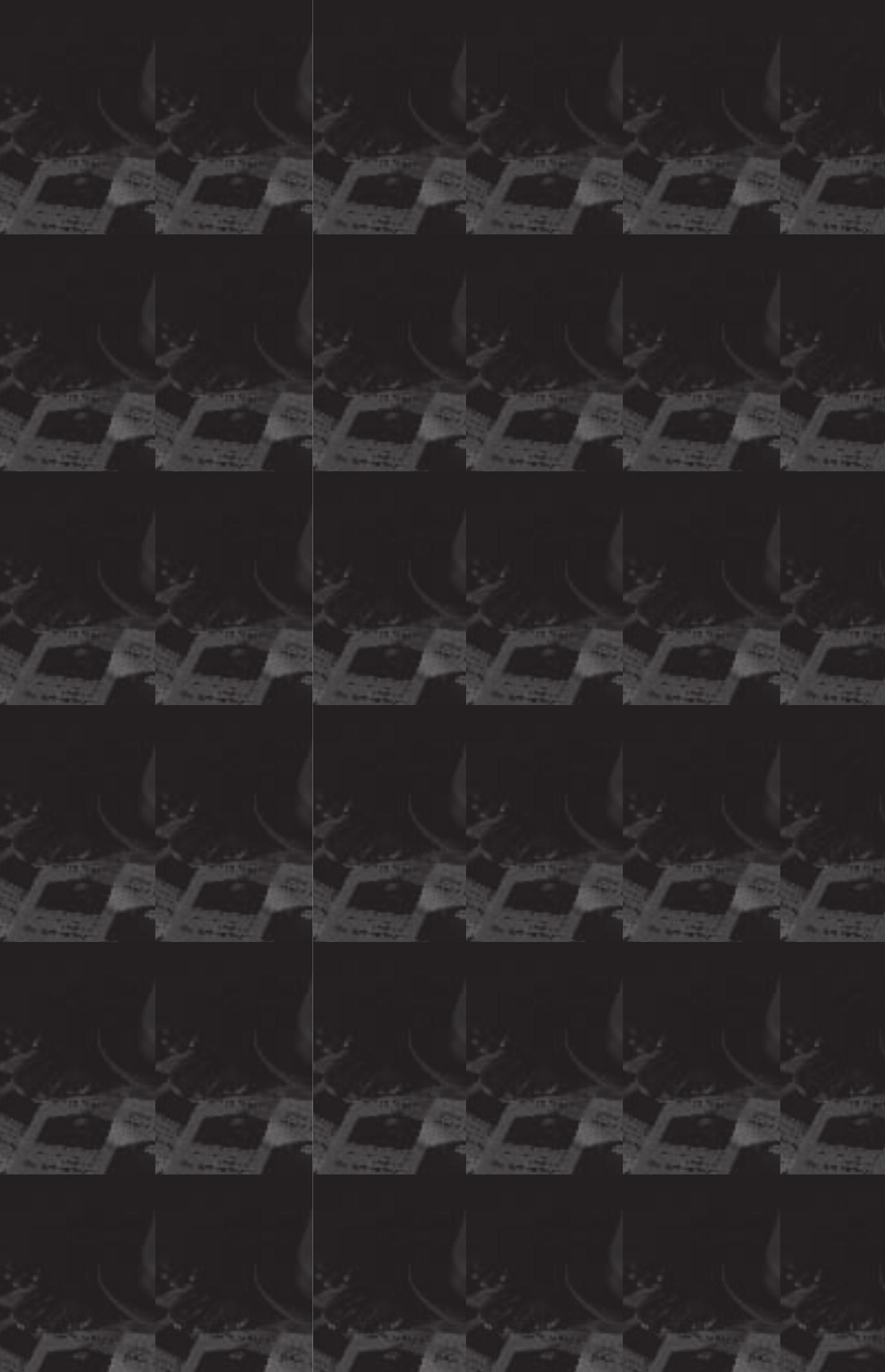

